\title{
A Case of Sinonasal Lymphoepithelial Carcinoma Presenting as a Palatal Swelling
}

\author{
Nikolaos Kolomvos ${ }^{1}$, Dionysios Kyrmizakis ${ }^{2}$, Evangelia Piperi ${ }^{3}$ and \\ Evanthia Chrysomali ${ }^{3 *}$ \\ ${ }^{1}$ Department of Oral and Maxillofacial Surgery, School of Dentistry, National and \\ Kapodistrian University of Athens, Greece \\ ${ }^{2}$ Otorhinolaryngology Head and Neck Surgery, Heraklion Hospital, Greece \\ ${ }^{3}$ Department of Oral Medicine and Pathology, School of Dentistry, National and \\ Kapodistrian University of Athens, Greece \\ *Corresponding Author: Evanthia Chrysomali, Department of Oral Medicine and \\ Pathology, School of Dentistry, National and Kapodistrian University of Athens, Greece. \\ DOI: $10.31080 /$ ASDS.2020.04.0886
}

Received: June 29, 2020

Published: July 25, 2020

(C) All rights are reserved by Evanthia

Chrysomali., et al.

\begin{abstract}
Lymphoepithelial carcinoma is a rare type of undifferentiated carcinoma, characterized by atypical epithelial cells surrounded by a reactive lymphoplasmacytic infiltrate. We report a case of sinonasal lymphoepithelial carcinoma arising in the left maxillary sinus in an 80-year-old Caucasian male patient. The lesion had invaded the adjacent lateral wall of the nasal cavity appearing on the palate as a painful swelling. There was no evidence of tumor involvement from the nasopharynx and larynx assessment and no swollen cervical lymph nodes were noticed on the head and neck imaging examination. An incisional biopsy from the oral cavity, as well as an endoscopic biopsy of the neighboring nasal mass were performed. The histopathological and immunohistochemical analysis of both specimens showed identical features and a common diagnosis of sinonasal lymphoepithelial carcinoma was rendered. In conclusion, oral swellings located on the palate may be associated with maxillary sinus malignancies. Thus, the clinician should include in the differential diagnosis not only primary oral carcinomas, but also malignant neoplasms arising from neighboring anatomic sites. Keywords: Lymphoepithelial carcinoma; maxillary sinus; palate; sinonasal
\end{abstract}

\section{Abbreviations}

CT: Computed Tomography; IMRT: Intensity-Modulated Radiation Therapy; LEC: Lymphoepithelial Carcinoma; MRI: Magnetic Resonance Imaging; SNUC: Sinonasal Undifferentiated Carcinoma

\section{Introduction}

Sinonasal malignancies are uncommon entities comprising less than $1 \%$ of all malignant neoplasms and about $3 \%$ of head and neck cancers [1]. Lymphoepithelial carcinoma (LEC) is defined by the World Health Organization (WHO) as a poorly differentiated or histologically undifferentiated carcinoma accompanied by a prominent reactive lymphoplasmacytic infiltrate, morphologically similar to non-keratinizing undifferentiated nasopharyngeal carcinoma [1]. LEC may arise in several head and neck locations, including maxillary sinus [2-4], ethmoid sinus [5] lower lip [6] soft palate and uvula [4] oral cavity [4] and salivary glands [7]. In addition, LEC can also develop in other sites of the body [8], and synonym terms have been used to describe the cases located outside of the pharyngeal region, such as lymphoepithelial-like [6] or lymphoepithelioma-like [5,8] carcinoma.
The occurrence of LEC involving the sinonasal tract is generally rare. According to recent systematic reviews [4,9], a total of 48 LEC cases that originating from the maxillary sinus and nasal cavity have been published in the English literature from 1980 to 2016. The sinonasal LECs encompassed a percentage of about $36 \%$ of the 112 total cases of oral, oropharyngeal, nasal and paranasal LECs [4]. This carcinoma is mostly prevalent in China, South East Asia, Northern Africa and affects more commonly males of their fifth to seven decade with a median age of 60 years. Environmental factors, tobacco smoking and genetic susceptibility have not been verified directly in the etiology of LEC $[1,10]$. A strong relationship with Epstein-Barr virus (EBV) is referred [1,10], although some EBV-negative cases have been described $[4,7]$. The EBV has been detected by in situ hybridization in the majority (87.5\%) of cases [4] of the sinonasal LEC $[10,11]$. LEC diagnosis requisites the nasopharynx examination in order to exclude loco-regional spread from a primary nasopharyngeal carcinoma [1-3]. Nasal epistaxis and obstruction are among the relatively frequent reported symptoms in LEC, but they are non-specific [4]. The clinical and imaging features of LEC are not pathognomonic making a preoperative diagnosis of this rare malignancy difficult. Herein, we present an interesting case of sinonasal lymphoepithelial carcinoma that was developed 
in the maxillary sinus and appeared initially as an oral swelling protruding from the palate. Our patient represents the $49^{\text {th }}$ case in the literature during a 36-year time period.

\section{Case Report}

An 80-year-old Caucasian male non-smoker, non-drinker patient presented at the Oral Medicine Clinic of the School of Dentistry complaining that his upper removable partial denture could not fit right for the last 2-months, due to a lump in his mouth. The medical history was not remarkable with no systemic diseases except of hypertension. The laboratory blood tests including examination of diabetes mellitus were within normal rates. The patient referred occasional incidents of nose epistaxis, which was apparent at the time of examination. (Figure 1A). On intraoral examination a firm and painful on palpation tumor mass covered by normal oral mucosa was observed on the palatal middle line extending to the left hard palate. A diffuse swelling of the adjacent edentulous alveolar mucosa, as well as displacement of the upper left incisors and canine were also noticed. (Figure 1A). The panoramic radiograph showed expansion of the adjacent left sinus as well as maxillary bone resorption, (Figure 1B). An ENT examination was performed by flexible endoscopy, which revealed a mass in the left nasal choanae, while the rest of the head and neck clinical examination was unremarkable. The imaging exploration by a CT scan and MRI revealed similar findings. A mass sized $16 \mathrm{X} 12 \mathrm{~mm}$ was observed in the left maxillary antrum with evidence of erosion and focal bone destruction of the lateral wall of the sinus. The lesion was extending within the anterior part of the adjacent nasal cavity (Figure 1C).

There was no evidence of tumor involvement from the nasopharynx and larynx assessment and no swollen cervical lymph nodes were noticed on the head and neck imaging examination. Based on the clinical findings and imaging features, malignant tumors originating from the nose and paranasal sinuses were considered in the differential diagnosis. A possible subtype of sinonasal squamous cell carcinoma or adenocarcinoma, lymphoma, lymphoepithelial carcinoma, or other primary malignancies or metastatic neoplasm from other site of the body were included in the list of the provisional clinical diagnoses. An incisional biopsy from the oral cavity and an endoscopic biopsy of the nasal mass (Figure 2) using straight biopsy forceps under local anesthesia with anesthetic support were performed. The histopathologic examination revealed identical features in both specimens, which exhibited irregular islands of large pleiomorphic cells with indistinct cell margins (Figure 3A and 3B). The neoplastic cells had oval or round vesicular nuclei with prominent nucleoli demonstrating a syncytial appearance, whereas a dense lymphocytic infiltrate was observed among the tumor islands (Figure 3B). Immunohistochemical investigation was performed and the neoplastic cells were found to be positive for CK19 (Figure 4A), pancytokeratin (panCK) (Fig-

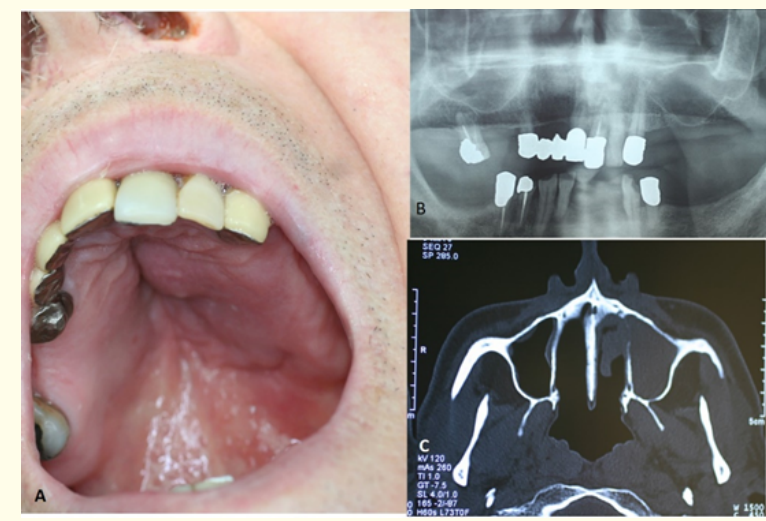

Figure 1: Clinical appearance of the palatal tumor at the initial presentation of the patient (A). The radiological image shows the thin residual alveolar ridge on the left edentulous maxilla (B). Computed tomography image. The left maxillary antrum is occupied by a mass which is extended into the nasal cavity (C).

ure 4B), keratins CK5/6 (Figure 4C), CK7, and EMA. The presence of EBV in tumor cells was confirmed by the in-situ hybridization method for EBV encoded early RNA (EBER). The lymphoid cells (Figure 4D) were positive for LCA (common leucocyte antigen). Based on the above findings a same diagnosis of lymphoepithelial carcinoma was rendered for both biopsies (nasal and oral).

The patient was referred for further investigation and treatment. The CT scans of the chest, abdomen, and pelvis showed no evidence of primary malignancy or distant metastases and the tumor was accordingly classified as pT2 [1]. A surgical approach with complete tumor resection and wide margins was not considered curable, due to the patient's age. Concurrent chemoradiotherapy was performed by using IMRT technique (cumulative dose $70 \mathrm{~Gy}$,

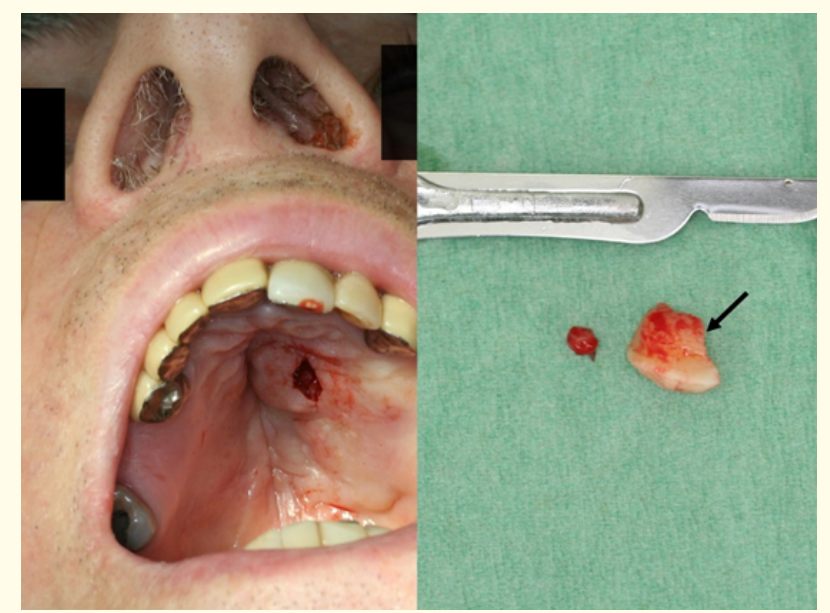

Figure 2: Left: Intraoperative photograph taken during the incisional oral biopsy. Right: The nasal and oral (arrow) surgical specimens respectively. 


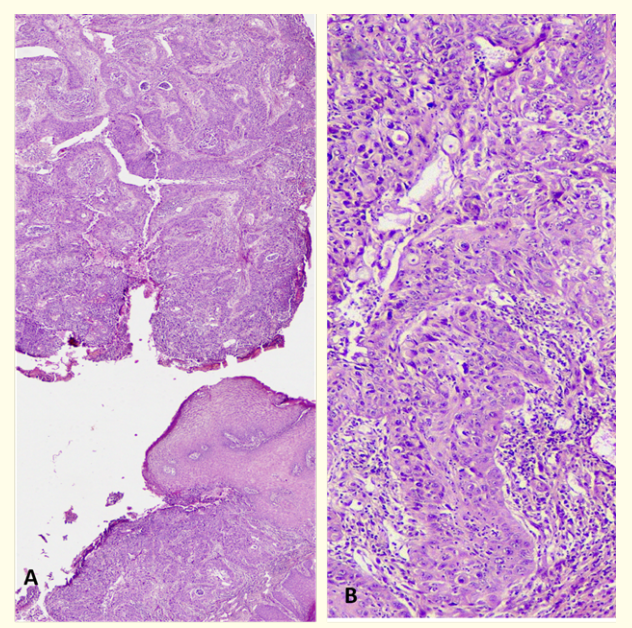

Figure 3: Histologic microphotograph showing tumor islands of neoplastic cells (A) that demonstrate vesicular nuclei (B) and lymphocytic infiltrate. (Hematoxylin and eosin stain, A X 250, B X 400).

delivered with a dose of 2.0 Gy five times a week) combined with chemotherapy (5-fluorouracil and cisplatin). He responded satisfactorily to the treatment and had remained disease-free for the next 24 months follow up without any tumor recurrence by MRI examination and clinical evaluation.

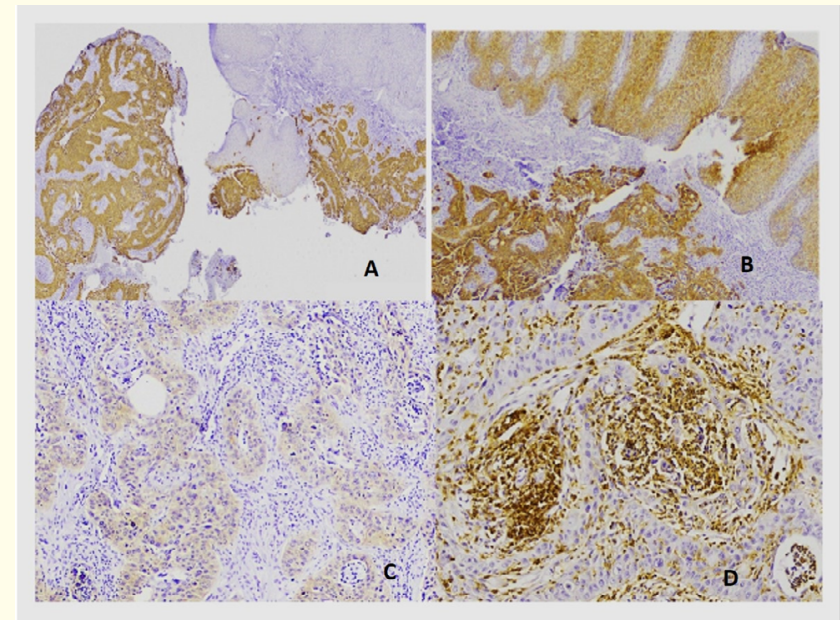

Figure 4: The neoplastic cells exhibit intense immunohistochemical staining (A) for CK19, (B) for pankeratin, (C) for CK 5/6, whereas the lymphoid cells (D) are positive for LCA. Immunohistochemical staining A and B X 250, C and D X400).

\section{Discussion}

The sinonasal cavities may be affected from various types of tumors predominantly of epithelial origin. The majority of the sinonasal malignant tumors arise in the maxillary sinus $(60 \%)$ followed by the nasal (30\%) cavity [13]. The clinical findings and symptoms vary according to the type, location and stage of malignancy. In early stages a maxillary sinus tumor may be asymptomatic or may cause mild unspecific signs and symptoms, such as headache, nasal obstruction or facial pain mimicking chronic inflammation of the sinus [2]. The tumor mass may be completely silent until it reaches sufficient size to interfere with surrounding structures. Symptoms may include epistaxis, postnasal discharge or rarely oral bulging. Unilateral hearing loss due to serous otitis media and paralysis of cranial nerves III, VI, IX, X, XI can be found in advanced stages of sinonasal carcinomas [12]. In our case, the cause of the patient's visit was a poor fitting denture, due to the presence of a palatal tumor. The extent of the diffuse swelling accompanied by teeth displacement were suggestive of a malignant tumor. However, developmental odontogenic cysts or locally aggressive odontogenic tumors may also appear clinically as large in size jaw expansions or soft tissue growths, especially when the lesions get secondarily inflamed and erode the cortical plates or in cases of destructive malignant jaw lesions. This possibility was excluded based on the panoramic radiograph examination, since there was no evidence of an osteolytic bone lesion in the maxilla. The left sinus expansion and maxillary bone thinning combined with the presence of the clinical sign of nasal epistaxis at the same side, were considered as indicative of sinonasal involvement. The diagnostic approach in order to investigate the exact location and extension of the lesion included both CT and MRI imaging.

The main histopathological differential diagnosis of sinonasal LEC is the sinonasal undifferentiated carcinoma (SNUC). SNUC and LEC may share similar morphologic features, although they represent distinct entities [11]. The presence of lymphoplasmacytic infiltrate in the stroma of LEC may be not a reliable criterion to distinguish LEC from SNUC [3]. Histologically, SNUC is characterized by nuclear pleiomorphism, numerous atypical mitotic figures and the presence of extensive necrosis. The tumor cells in LEC exhibit lightly eosinophilic cytoplasm, vesicular or plump nuclei with prominent nucleoli and indistinct cell borders, resulting in a syncytial appearance. Necrosis and keratinization are usually not evident. Despite the unclear etiology of both carcinomas, EBV involvement in SNUC seems unlikely, since EBV usually is not detected in the tumor cells, in contrast to sinonasal LEC, which is positive for EBV in most cases. The different immunoprofile of SNUC is also contributory to the differential diagnosis, since in contrast to LEC, the tumor cells in SNUC do not show immunoreactivity for CK4, CK5/CK6 and CK14 [1].

The importance of the correct diagnosis plays an essential role, due to the differences in the biological behavior between these two malignancies. SNUC is a highly aggressive neoplasm demonstrating a more destructive local invasiveness of the adjacent anatomic 
structures, tendency for lymph node and distant metastases and a less favor prognosis compared to LEC. A standard treatment protocol approach has not been established, given the rarity of these malignancies, the anatomic complexity of the region and the proximity with structures of clinical importance, such as the orbit and brain. The therapeutic strategies have revolved around surgery $[2,9]$, chemotherapy [5] and radiotherapy [3,5,9,12]. A surgical approach sometimes may be not possible depending on the size of the tumor, the medical history and age of the patient, such as in our case. Chemotherapy can be used before, concurrent with, or as an adjuvant to precision radiotherapy. The concomitant radiotherapy and chemotherapy increase survival and enables locoregional control [12]. Prognosis of LEC is better than other head and neck carcinomas, since regardless of the disease stage the 5-year overall survival is estimated at $70 \%$ and disease-specific survival at $78 \%[14]$.

\section{Conclusion}

Palatal swellings may rarely be associated with sinonasal malignancy. An oral tumor mass that cannot be attributed to inflammatory or other local etiological factors requires the patient's evaluation including a detailed clinical examination of the oropharynx, maxillofacial region and a complete nose and throat exploration. The early recognition of LEC is essential considering the proximity of the nasal cavities and paranasal sinuses with essential anatomic structures, such as orbit, base of the skull and the tendency of the maxillary sinus carcinomas for local spread, as well as metastasis.

\section{Conflict of Interest}

The authors declare that they have no financial or any conflict of interest

\section{Bibliography}

1. Franchi A., et al. "Data Set for the Reporting of Carcinomas of the Nasal Cavity and Paranasal Sinuses: Explanations and Recommendations of the Guidelines from the International Collaboration on Cancer Reporting". Archives of Pathology and Laboratory Medicine 143. 4 (2019): 424-431.

2. Mohammed D., et al. "Lymphoepithelial carcinoma in the maxillary sinus: a case report”. Journal of Medical Case Report 6.416 (2012): $1-4$

3. Muthayam SR., et al. "Lymphoepithelial carcinoma arising from the maxillary antrum: a clinicopathological report of a rare lesion in an unusual site". Quantitative Imaging in Medicine and Surgery 4.6 (2014): 512-515.

4. Rytkönen $\mathrm{AE}$., et al. "Lymphoepithelial carcinoma: two case reports and a systematic review of oral and sinonasal cases". Head and Neck Pathology 5 (2011): 327-334.
5. Hajiioannou JK., et al. "Nasopharyngeal-type undifferentiated carcinoma (lymphoepithelioma)". Journal of Otolaryngology 35. 2 (2006): 147-151.

6. Almeida LY., et al. "EBV-negative lymphoepithelial-like carcinoma of the lower lip". Autopsy Case Report 10 (2020): e138.

7. Zhan KY., et al. "Lymphoepithelial carcinoma of the major salivary glands: Predictors of survival in a non-endemic region". Oral Oncology 52. (2016): 24-29.

8. Han AJ., et al. "Lymphoepithelioma-like carcinoma of the lung with a better prognosis. A clinicopathologic study of 32 cases". The American Journal Clinical Pathology 115.6 (2001): 841850.

9. Takakura H., et al. "Lymphoepithelial carcinoma of the maxillary sinus. A case report and review of the literature". Medicine 97.28 (2018): e11371.

10. Zong Y., et al. "Epstein-Barr virus infection of sinonasal lymphoepithelial carcinoma in Guangzhou". Chinese Medical Journal 114.2 (2001): 132-136.

11. Jeng YM., et al. "Sinonasal undifferentiated carcinoma and nasopharyngeal-type undifferentiated carcinoma: two clinically, biologically, and histopathologically distinct entities". American Journal of Surgical Pathology 26.3 (2002): 371-376.

12. Llorente JL., et al. "Sinonasal carcinoma: clinical, pathological, genetic and therapeutic advances". Nature Reviews of Clinical Oncology 11.8 (2014): 460-472.

13. Lewis JS and Castro EB. "Cancer of the nasal cavity and paranasal sinuses". The Journal of Laryngology and Otology 86.3 (1972): 255-262.

14. Chan JYK., et al. "Non-nasopharyngeal head and neck lymphoepithelioma-like carcinoma in the United States: a populationbased study". Head and Neck 38.1 (2016): E1294-300.

\section{Assets from publication with us}

- Prompt Acknowledgement after receiving the article

- Thorough Double blinded peer review

- Rapid Publication

- Issue of Publication Certificate

- High visibility of your Published work

Website: www.actascientific.com/

Submit Article: www.actascientific.com/submission.php

Email us: editor@actascientific.com

Contact us: +919182824667 